\title{
Remote Sensing Image Compression based on Binary Tree and Optimized Truncation ${ }^{\underline{2}}$
}

\author{
Ke-Kun Huanga ${ }^{\mathrm{a}}$, Hui Liu', Chuan-Xian Ren, ${ }^{\mathrm{b}, *}$ Yu-Feng Yub ${ }^{\mathrm{b}}$, Zhao-Rong Lai ${ }^{\mathrm{c}}$ \\ ${ }^{a}$ School of Mathematics, JiaYing University, Meizhou, Guangdong, 514015, China. \\ ${ }^{b}$ Intelligent Data Center and Department of Mathematics, Sun Yat-Sen University, Guangzhou 510275, China. \\ ${ }^{c}$ Department of Mathematics, College of Information Science and Technology, JiNan University, Guangzhou 510632, China.
}

\begin{abstract}
The remote sensing image data is so vast that it requires compression by low-complexity algorithm on space-borne equipment. Binary tree coding with adaptive scanning order (BTCA) is an effective algorithm for the mission. However, for large-scale remote sensing images, BTCA requires a lot of memory, and does not provide random access property. In this paper, we propose a new coding method based on BTCA and optimize truncation. The wavelet image is first divided into several blocks which are encoded individually by BTCA. According the property of BTCA, we select the valid truncation points for each block carefully to optimize the ratio of rate-distortion, so that a higher compression ratio, lower memory requirement and random access property are attained. Without any entropy coding, the proposed method is simple and fast, which is very suitable for space-borne equipment. Experiments are conducted on three remote sensing image sets, and the results show that it can significantly improve PSNR, SSIM and VIF, as well as subjective visual experience.
\end{abstract}

Keywords: Remote sensing image compression, on-board compression, binary tree coding, rate-distortion optimization, Wavelet application

\section{Introduction}

Remote sensing images have wide applications such as object detection [1], environmental monitoring [2], and urban planning [3], etc. With the rapid development of sensor technology, high spatial resolution images are more easily acquired. Since the images will take up a great deal of storage space, and the hardware of spaceborne equipments is limited, great efforts are made to seek low-complexity image compression algorithms.

Because wavelet transform expresses the local timedomain and frequency-domain characterization and provides excellent muti-resolution analysis features, most of the state-of-the-art coding systems are based

\footnotetext{
This work is supported in part by National Science Foundation of China under Grants 61403164 and 61572536, in part by the Fundamental Research Funds for the Central Universities under Grant 161gzd16.

${ }^{*}$ Corresponding author

Email addresses: kkcocoon@163. com (Ke-Kun Huang), imlhxme163. com (Hui Liu), rchuanx@mail.sysu.edu.cn (Chuan-Xian Ren), yuyufeng220@163.com (Yu-Feng Yu), laizhrejnu.edu. cn (Zhao-Rong Lai)

Preprint submitted to Digital Signal Processing
}

on wavelet transform. The Embedded Zerotree Wavelet (EZW) [4] is the first successful compression algorith$\mathrm{m}$ based on wavelet transform, which constructs zerotree by the correlations across scales in each bit-plane, so that a large number of no-significant coefficients are predicted successfully by a root of zerotree. Set Partitioning in Hierarchical Tree (SPIHT) [5] is another famous algorithm, which can significantly improve the performance with lower complexity compared with EZW. In [6], the Set Partitioned Embedded Block (SPECK) coder was proposed to extend SPIHT.

EZW, SPIHT and SPECK exploit self-similarity across scales, while there are some algorithms that utilize the clustering characteristic within each subband. In [7], the QuadTree Coding (QTC) algorithm for wavelet image compression was proposed. It splits each node into four descendent nodes once it is significant with the current threshold. QTC produces an embedded data stream, supports quality scalability, and permits regionof-interest coding. The Embedded Block Coding with Optimized Truncation (EBCOT) algorithm [8], adopted in the JPEG2000 standard [9], combines layered block coding, rate-distortion optimization, and context-based

February 14, 2017 
arithmetic coding in an efficient and highly scalable way.

Recently, there are some methods which are specifically designed for remote sensing images. In [10], $\mathrm{Li}$ et al. proposed the two-dimensional oriented wavelet transform for remote sensing images based on JPEG2000. Kulkarni et al. [11] presented a scan-based JPEG2000 for large-scale images. However, JPEG2000 is too complex, which hinders JPEG2000 to be a compression standard for space-borne missions where the capacity of storage and transmission is limited.

In [12], the Consultative Committee for Space Data Systems (CCSDS) [13] published an image data compression standard (CCSDS-IDC) based on wavelet transform. The recommendation specifically targets space-borne missions, and focuses more on complexity and less on compression performance. It has a limited set of options, supporting its successful application without in-depth algorithm knowledge. In [14], some extensions of CCSDS-IDC have been proposed. In [15], the directional lifting wavelet transform was used to improve the performance of CCSDS-IDC.

Besides JPEG2000 and CCSDS-IDC, the other compression algorithms were also applied for remote sensing image. In [16], a modified listless strip based SPIHT was proposed to reduce system complexity and minimize processing time and memory usage. In [17], an improved SPIHT was proposed for multispectral image compression for various band images with high resolution. In [18], a three dimensional SPECK was proposed for hyperspectral image compression. In [19], a SAR complex image data compression algorithm based on QTC in wavelet transform domain was proposed, showing that QTC achieves the best performance for SAR complex image compression. In [20], the cubic B-spline fuzzy transform was proposed for an efficient and secure compression in wireless sensor networks.

Recently, compressed sensing has been successfully used in many fields of computer vision [21, 22], which also has been proved to perform well on image compression. In [23], a SAR image compression using multiscale dictionary learning and sparse representation was proposed, which is better for preserving the important features of SAR images with a competitive compression performance. In [24], an adaptive spectral-spatial compression of hyperspectral image with sparse representation has been proposed, which outperforms some state-of-the-art HSI compression methods in terms of the rate-distortion and spectral fidelity performances. For remote sensing image compression, the complexity of algorithm is very important. A new fast algorithm for image and video compression based on dis- crete Tchebichef transforms DTT that shows good performance [25]. The proposed DTT-based method is multiplication-free and only requires a reduced number of additions and bit-shifting operations, which is suitable for remote sensing image compression for its lower complexity.

In 2012, a fast remote sensing image coder, named binary tree coding with adaptive scanning order (BTCA) [26], was presented. It used the binary tree for coding remote sensing image in wavelet domain, and then developed an adaptive scanning order to traverse the binary tree, so that better performance and visual effect are attained. Unlike JPEG 2000 or CCSDS-IDC, BTCA is very fast because it does not use any entropy coding. BTCA is very easy to implement in hardware and very suitable for on-board compression. Since BTCA was proposed, some improvements have been developed. In [27], an embedded image compression based on fractal and wavelet is proposed, where the lowest frequency subband of wavelet domain is coded by fractal first, and the coding error and other sub-bands are coded by BTCA. In [28], a Human vision-based Adaptive Scanning (HAS) for the compression of remote sensing image was proposed, which generates an importance weighting mask according to the human visual characteristics before applying BTCA. In [29], a content-based adaptive scanning scheme was proposed for remote sensing image compression, which provides different scanning orders among and within subbands based on BTCA.

However, BTCA needs a lot of memory to store the binary tree for large-scale remote sensing images, and does not provide random access property. In [26], BTCA is processed with a scan-based mode, named BTCAS, to deal with this problem. Nevertheless, BTCAS sacrifices in compression efficiency. In this paper, we propose a new coding method based on Binary Tree and Optimized Truncation (BTOT). Compared with BTCA, the proposed BTOT method significantly improves the PSNR, while it requires less memory and has the random access property.

The main contributions of this paper are listed as follows:

- We propose an improved BTCA algorithm by optimized truncation. The wavelet image is first divided into several blocks which are encoded individually by BTCA. Then we apply rate-distortion optimization to get the higher compression ratio, less memory requirement and the random access property.

- We find that the distortion-rate almost decreases as the adaptive scanning level increases, so we record 
the bit rate and corresponding distortion as a candidate truncation point after each adaptive scanning level to get finer embedded bit-streams.

The remainder of the paper is organized as follows: In section 2, we introduce the BTCA algorithm briefly. In section 3, we describe the proposed method in detail. In section 4, we give the analyses about time complexity, memory requirement and the feature of the coding stream. The experimental results are presented in section 5. Finally, we provides the conclusion in section 6.

\section{The BTCA algorithm}

Because the coefficients in the high-frequency wavelet subbands are sparse, we can divide the subbands into several blocks and then check whether they contain significant coefficients. The tree structure is widely used in many fields of information sciences $[30,31,32]$, and is also a good model for compression [33]. In [7], each block is split into four sub-blocks once it tests as significant with respective to the curren$\mathrm{t}$ threshold. However, the binary tree is more efficient than quadtree [34], so the binary tree coding in wavelet domain is proposed in [26]. Because our method is based on BTCA, we briefly describe BTCA as follows.

Suppose a wavelet image is of size $2^{N} \times 2^{N}$. We first transform it into an one-dimensional vector using morton scanning order, denoted by $V$. Then we construct the binary tree $\Gamma(t)$ for $1 \leq t<2 \times 2^{N} \times 2^{N}$. The bottom level of the binary tree consists of the absolute value of each coefficient:

$\Gamma(t)=\left|V\left(t-2^{N} \times 2^{N}\right)\right|$ for $2^{N} \times 2^{N} \leq t<2 \times 2^{N} \times 2^{N}$.

The upper levels of the tree are defined iteratively:

$\Gamma(t)=\max \{\Gamma(2 t), \Gamma(2 t+1)\}$ for $1 \leq t<2^{N} \times 2^{N}$.

After constructing the binary tree, we can traverse the tree by depth-first, which can be expressed as a function code $=B T C\left(\Gamma, t, T_{k}\right)$ as Algorithm 1, where $t$ is a tree node index of the binary tree, and $T_{k}$ is a threshold where $T_{0}=2^{\left\lfloor\log _{2} \Gamma(1)\right\rfloor}$ and $T_{k}=T_{0} / 2^{k}$.

The coefficients on the edges are often large, so we can scan the neighbor of the previous significant coefficients before other regions are scanned. BTCA uses an adaptive scanning order, namely, from the bottom level to the top, if a coefficient's brother is a previous significant coefficient, then we traverse the coefficient and its descendants by depth-first. Based on Algorithm 1, the detail steps of BTCA can be described as a function code $=B T C A\left(\Gamma, T_{k}\right)$ as Algorithm 2.

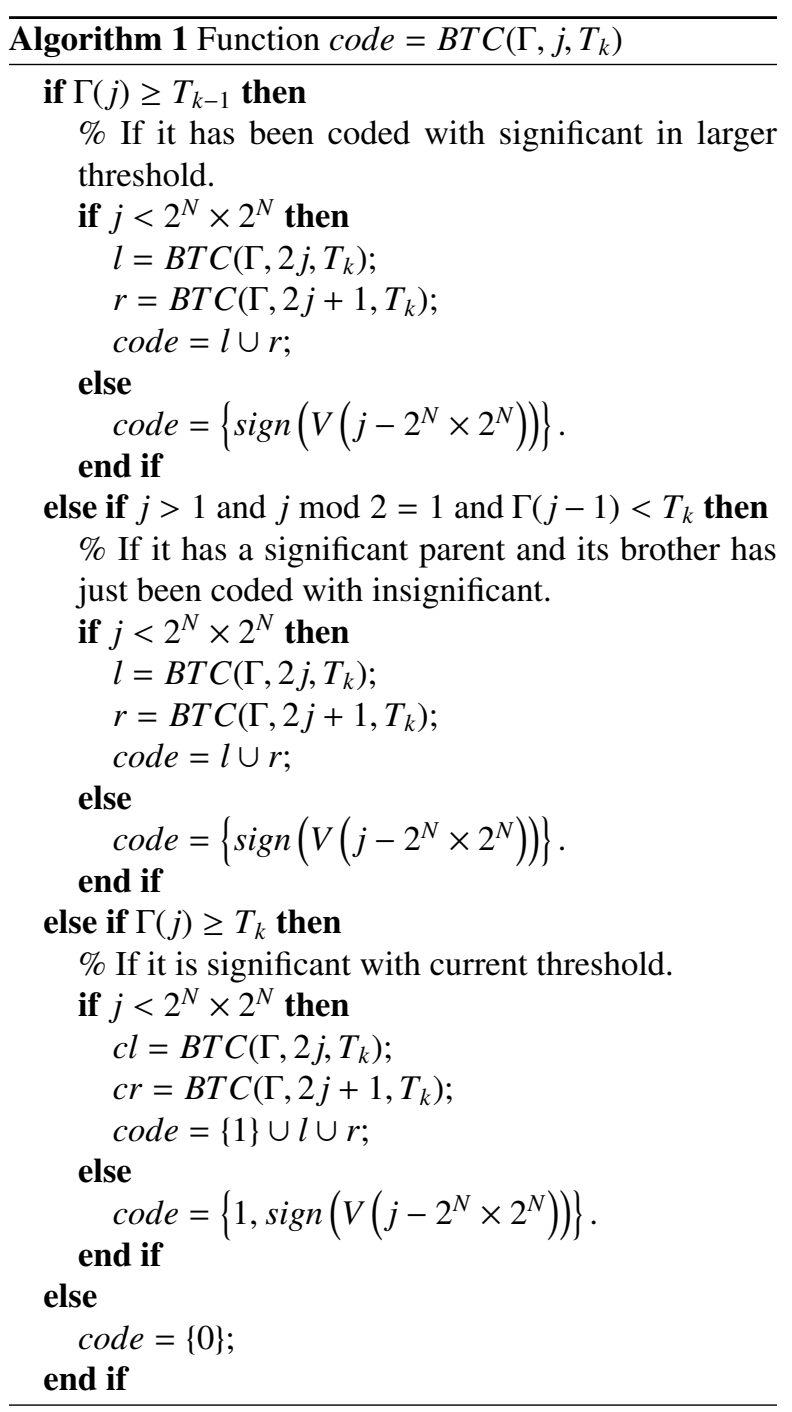




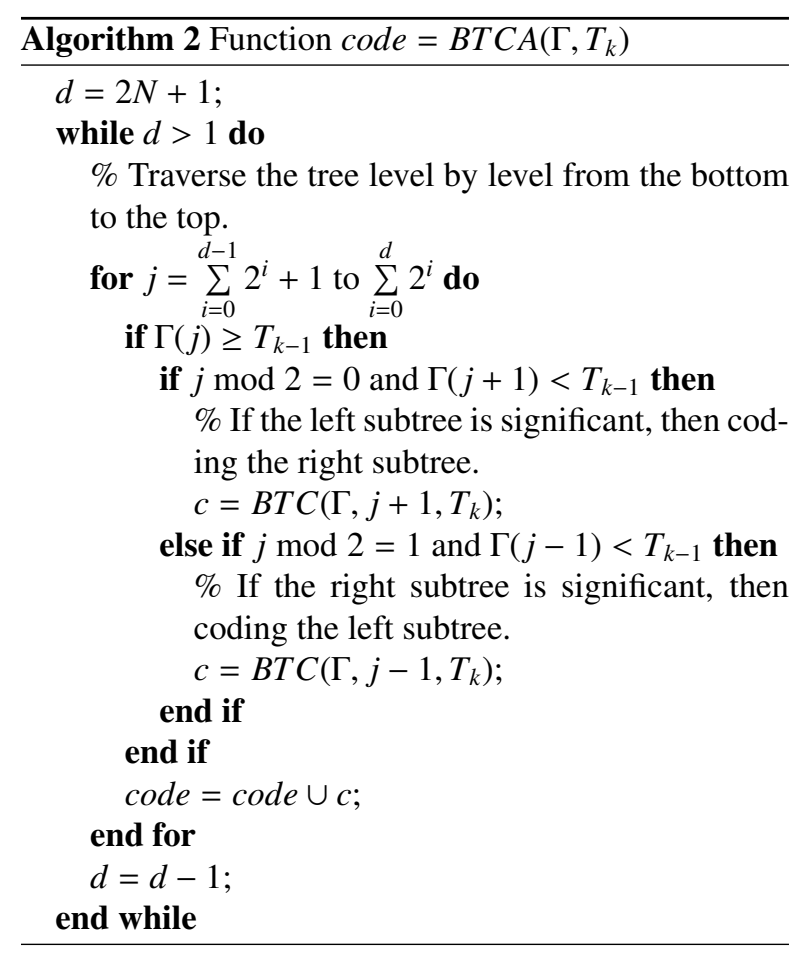

\section{Compression based on binary tree and optimized truncation}

BTCA achieves state-of-the-art performance, but it needs a lot of memory to store the binary tree for a large remote sensing image, and does not provide random access property. In this section, we propose a new method based on binary tree and optimized truncation, in order to get higher compression ratio and less memory requirement, as well as the random access property.

The wavelet image is first divided into several blocks. Then the blocks are encoded by BTCA independently. To apply rate-distortion optimization, the most important step is how to get the valid truncation points. We find that the distortion-rate almost decreases as the adaptive scanning level increases, so we can record the bit rate and corresponding distortion as a candidate truncation point after each adaptive scanning level. The number of the proposed candidate truncation points is more than EBCOT [8] in which there are only 4 candidate truncation points for each bit-plane. The detail process can be described as follows.

Suppose the bit rate $R_{j}$ and the corresponding distortion $D_{j}$ at the $j^{\text {th }}$ candidate truncation point of a block are obtained. Let

$$
S_{j}=\frac{D_{j-1}-D_{j}}{R_{j}-R_{j-1}}
$$

denote the local distortion-rate. We hope that $S_{j}$ decreases as $j$ increases. Figure 1 shows the variation of $S_{j}$ when using the proposed method to encode a block of a remote sensing image with four bit-planes whose size is $2^{6} \times 2^{6}$. Except the reduplicative truncation points, there are 30 candidate truncation points in the proposed method, while there are $1+4 \times 3=13$ points in EBCOT [8]. So the proposed method can achieve finer embedded bit-streams than EBCOT.

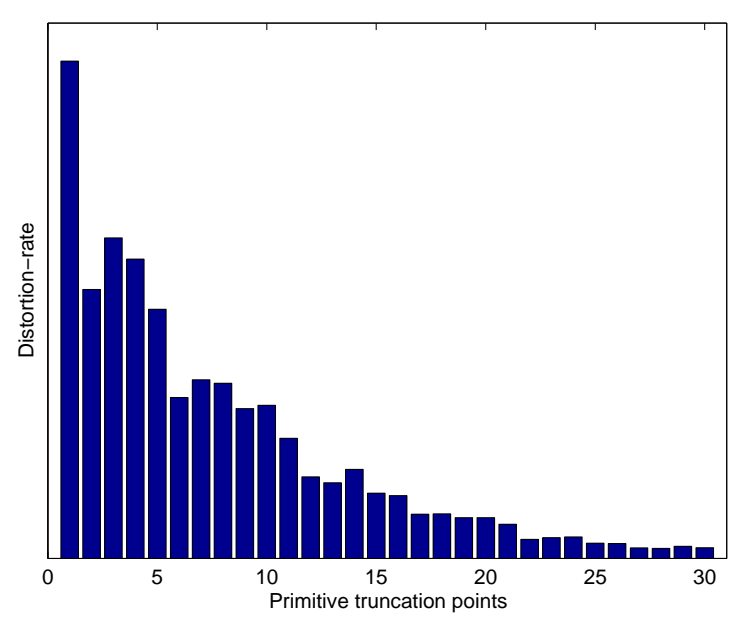

Figure 1: Distortion-rates of the candidate truncation points of the proposed method.

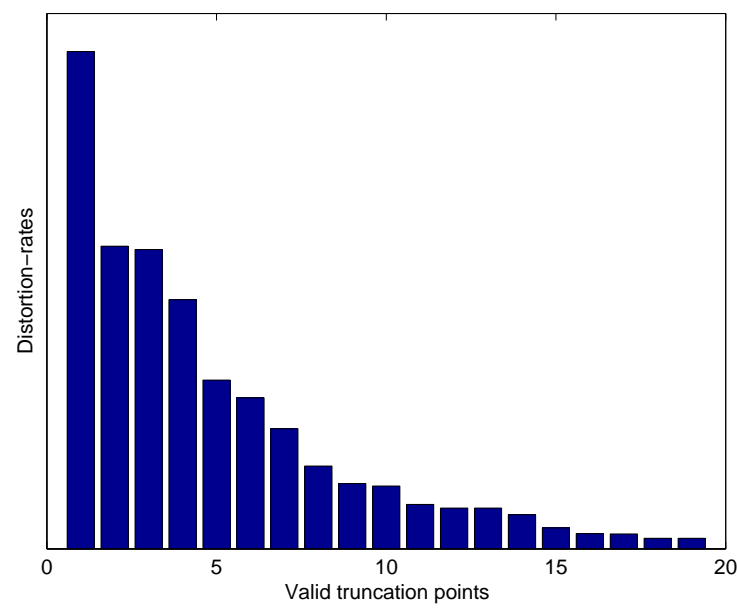

Figure 2: Distortion-rates of the valid truncation points of the proposed method.

We use the Post-Compression Rate Distortion (PCRD) optimization algorithm [8] to select the candidate truncation points of the independent block such 
that the distortion is minimized at a specified bit rate. If $S_{j+1}>S_{j}$, then $j^{\text {th }}$ candidate truncation point cannot be selected, and we need to update

$$
S_{j+1}=\frac{D_{j-1}-D_{j+1}}{R_{j+1}-R_{j-1}} .
$$

Then we compare it with $S_{j-1}$. If $S_{j+1}>S_{j-1}$, then $(j-1)^{\text {th }}$ candidate truncation point cannot be selected, and we need to update

$$
S_{j+1}=\frac{D_{j-2}-D_{j+1}}{R_{j+1}-R_{j-2}} .
$$

This process is performed until $S_{j+1}<S_{j_{k}}$, where

$$
S_{j+1}=\frac{D_{j_{k}-1}-D_{j+1}}{R_{j+1}-R_{j_{k}-1}} .
$$

We can select the strictly decreasing $\left\{S_{j_{k}}\right\}$ as a series of valid truncation points. Figure 2 shows distortionrates of the valid truncation points of the candidate truncation points in Figure 1. Because $S_{j}$ is almost monotonically decreasing, it remains most of the candidate truncation points in Figure 2. Using PCRD optimization algorithm, a final truncation point can be attained for each block at a specified rate. The number of the final truncation points is equal to the number of blocks, so there are only a little extra storage to record the truncation points. Applying the rate-distortion optimization, the proposed method gets better performance and requires less memory, as well as random access property.

The proposed method based on Binary Tree and Optimization Truncation is called BTOT. The detail encoding algorithm of the proposed method can be summarized as Algorithm 3.

Algorithm 3 The detail encoding algorithm of the proposed BTOT method.

Input: The original image $I$; The coding length bits.

Output: The encoded information code and the code length of each block $R_{t}$.

1: Perform wavelet transform: $I_{w}=D W T(I)$;

2: Divide $I_{w}$ into blocks $\left\{B_{m}, m=1, \cdots, M\right\}$ where $B_{m}$ is of size $2^{n} \times 2^{n}$;

3: \% Coding each block alone as follows.

4: for $m=1$ to $M$ do

5: $\quad$ Construct binary tree $\Gamma_{m}$ for $B_{m}$;

6: Initialize truncation points $R_{m}=\{\}$ and $D_{m}=\{\}$.

7: $\quad$ Traverse the binary tree by depth-first with $T_{0}$ :

$$
c_{m}=B T C\left(\Gamma_{m}, 1, T_{0}\right) \text {; }
$$

Record truncation points: $R_{m}=\left\{R_{m}, r\right\}$ and $D_{m}=$ $\left\{D_{m}, M S E\right\}$, where $r=$ length $\left(c_{m}\right)$ and $M S E$ denotes the mean square errors between original image and the image reconstructed by the current code $c_{m}$.

9: $\%$ Coding with each threshold $T_{k}$ as follows.

10: $\quad$ for $k=1$ to $K$ do

$\%$ Traverse the tree level by level from the bottom to the top.

$$
\begin{aligned}
& \text { for } j=\sum_{i=0}^{d-1} 2^{i}+1 \text { to } \sum_{i=0}^{d} 2^{i} \text { do } \\
& \text { if } \Gamma_{m}(j) \geq T_{k-1} \text { then } \\
& \quad \text { if } j \bmod 2=0 \text { and } \Gamma_{m}(j+1)<T_{k-1} \\
& \quad \text { then }
\end{aligned}
$$

$\%$ If the left subtree is significant, then coding the right subtree.

$$
c=B T C\left(\Gamma_{m}, j+1, T_{k}\right) \text {; }
$$

else if $j \bmod 2=1$ and $\Gamma_{m}(j-1)<$ $T_{k-1}$ then

$\%$ If the right subtree is significant, then coding the left subtree.

$$
c=B T C\left(\Gamma_{m}, j-1, T_{k}\right)
$$

$$
\text { end if }
$$

\section{end if}

$$
c_{m}=c_{m} \cup c \text {; }
$$

\section{end for}

$$
d=d-1
$$

Record truncation points;

\section{end while}

Perform magnitude refinement pass and record truncation points;

32: Initialize $R_{v}=\{\}, S_{v}=\{\}$. \% Valid truncation points of all blocks.

33: for $m=1$ to $M$ do

34: Initialize $D=\{0\}, R=\{0\}, S=\{\infty\}, j=1 ; \%$ Valid truncation points of the current block.

35: $\quad$ for $i=1$ to length $\left(R_{m}\right)$ do

36: $\quad S_{t}=\frac{D(j)-D_{m}(i)}{R_{m}(i)-R(j)}$

$$
\text { 37: } \quad \text { while } S_{t}>S(j) \text { do }
$$

$$
\text { 38: } \quad j=j-1 \text {; }
$$

$$
S_{t}=\frac{D(j)-D_{m}(i)}{R_{m}(i)-R(j)} ;
$$

end while

$j=j+1$;

$D(j)=D_{m}(i)$;

$R(j)=R_{m}(i)$;

$S(j)=S_{t}$;

$$
\text { end for }
$$


46: $\quad$ Delete the first element in $R$ and $S$, and append some zeros to $R$ and $S$ such that length $(R)=L$ and length $(S)=L$;

47: $\quad R_{v}=R_{v} \cup R ; S_{v}=S_{v} \cup S$;

48: end for

49: Sort $S_{v}$ in descending order and get the permutation vector $S_{\text {ind }}$ of the corresponding element;

50: Initialize $R_{t}(m)=0$ for $m=1, \cdots, M$;

51: $i=1$;

52: while $\sum_{m}\left(R_{t}(m)\right)<$ bits do

53: $\quad s_{i}=S_{\text {ind }}(i)$;

54: $\quad m=\bmod \left(s_{i}, L\right) ;$

55: $\quad R_{t}(m)=R_{v}\left(s_{i}\right)$;

56: $\quad i=i+1$;

57: end while

58: Initialize code $=\{\}$;

59: for $m=1$ to $M$ do

60: $\quad$ code $=$ code $\cup c_{m}\left(1: R_{t}(m)\right)$;

61: end for

To better illustrate the proposed method, we give a simple example. Suppose that there is a $4 \times 4$ block whose coefficients are shown in the left side of Figure 3. The block is transformed into one-dimensional vector with morton scanning order, resulting $V=$ $\{10,5,4,3,3,1,2,5,0,0,1,1,2,3,6,3\}$. Then we can construct the binary tree according to Eq. (1) and Eq. (2), as shown in the right side of Figure 3. Table 1 shows the candidate truncation points for coding the block. We initialize the distortion by $D_{0}=\sum_{i, j} x_{i, j}^{2}=249$, the rate $R_{0}=0$ and the distortion-rate $S_{0}=$ inf. Then we first traverse the binary tree by depth-first with $T=8$. The resulting code is " 1111100000 ", and the reconstructed coefficients are $12,0,0,0,0,0,0,0,0,0,0,0$, $0,0,0,0$, respectively, so $R_{1}=10, D_{1}=153$ and $S_{1}=\frac{D_{0}-D_{1}}{R_{1}-R_{0}}=9.6$.

Then we traverse the binary tree from bottom level to top level with $T=4$. For each level, if the brother of a coefficient is significant with $T=8$, then we prefer to traverse the coefficient and its descendants, i.e., with $T=4$, we perform $B T C(\Gamma, 17,4)$ for level=4, $B T C(\Gamma, 9,4)$ for level $=3, B T C(\Gamma, 5,4)$ for level $=2$ and $B T C(\Gamma, 3,4)$ for level=1 in turn. After traversing with $T=4$, we perform magnitude refinement pass for all the previous significant coefficients, as No. 6 candidate truncation point in Table 1.

With $T=2$, we perform $\operatorname{BTC}(\Gamma, 19,2)$, $B T C(\Gamma, 22,2)$ and $B T C(\Gamma, 31,2)$ for level=4, $B T C(\Gamma, 10,2)$ and $B T C(\Gamma, 14,2)$ for level=3, $\operatorname{BTC}(\Gamma, 6,2)$ for level=2 in turn, i.e., we traverse the brothers of the yellow coefficients in Figure 3 first. After traversing with $T=4$, we get 10 candidate truncation points, as shown in Table 1.

After the candidate truncation points are attained, we need to calculate the valid truncation points. Because $S_{2}>S_{1}$, the No. 1 candidate truncation point cannot be selected as a valid one, and we need to update $S_{2}=$ $\frac{D_{0}-D_{2}}{R_{2}-R_{0}}=\frac{249-129}{12-0}=10$. Because $S_{4}>S_{3}$, we need to update $S_{4}=\frac{D_{2}-D_{4}}{R_{4}-R_{2}}=\frac{129-93}{21-12}=4$. This process can be repeated and we can get all the valid truncation points, as shown in Table 2.

Table 1: The candidate truncation points of the proposed method for the example in Figure 3.

\begin{tabular}{cccrrr}
\hline No. & $T$ & Level & $D$ & $R$ & $S$ \\
\hline 0 & - & - & 249 & 0 & inf \\
1 & 8 & - & 153 & 10 & 9.6 \\
2 & 4 & 4 & 129 & 12 & 12.0 \\
3 & 4 & 3 & 129 & 13 & 0.0 \\
4 & 4 & 2 & 93 & 21 & 4.5 \\
5 & 4 & 1 & 57 & 27 & 6.0 \\
6 & 4 & 0 & 53 & 28 & 4.0 \\
7 & 2 & 4 & 32 & 34 & 3.5 \\
8 & 2 & 3 & 23 & 39 & 1.8 \\
9 & 2 & 2 & 11 & 45 & 2.0 \\
10 & 2 & 0 & 8 & 50 & 0.6 \\
\hline
\end{tabular}

Table 2: The valid truncation points of the proposed method for the example in Figure 3.

\begin{tabular}{cccrrr}
\hline No. & $T$ & Level & $D$ & $R$ & $S$ \\
\hline 0 & - & - & 249 & 0 & inf \\
2 & 4 & 4 & 129 & 12 & 10.0 \\
5 & 4 & 1 & 57 & 27 & 4.8 \\
6 & 4 & 0 & 53 & 28 & 4.0 \\
7 & 2 & 4 & 32 & 34 & 3.5 \\
9 & 2 & 2 & 11 & 45 & 1.9 \\
10 & 2 & 0 & 8 & 50 & 0.6 \\
\hline
\end{tabular}

\section{Analysis and discussion}

\subsection{Time complexity}

Suppose an entire wavelet image is of size $2^{N} \times 2^{N}$, and the blocks are of size $2^{n} \times 2^{n}$, where $N=p n$. There are $2^{p} \times 2^{p}=2^{2 p}$ blocks. For each block, there are $2^{n} \times 2^{n}=2^{2 n}$ coefficients consisting the bottom level of the binary tree. Constructing a tree node of upper level requires a comparison with two descendants. Let 


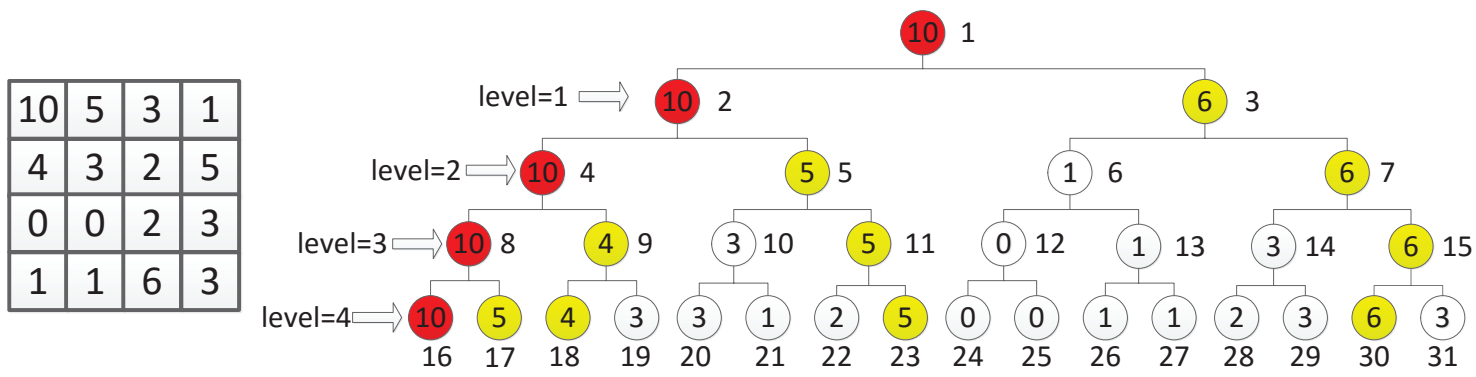

Figure 3: A simple example to illustrate the proposed method. Left: a block to be encoded. Right: The corresponding binary tree.

$D=\log _{2}\left(2^{2 n}\right)+1=2 n+1$ stands for the tree depth, then the comparison times for constructing all the trees of BTOT is:

$$
\begin{aligned}
C_{\text {BTOT }} & =2^{2 p} 2^{2 n}\left(\frac{1}{2}+\frac{1}{2^{2}}+\cdots+\frac{1}{2^{D-1}}\right) \\
& =2^{2 p} 2^{2 n}\left(1-\frac{1}{2^{2 n}}\right) \\
& =2^{2 p}\left(2^{2 n}-1\right) \\
& =\left(2^{2 N}-2^{2 p}\right) .
\end{aligned}
$$

While the comparison times for constructing the binary tree of BTCA is:

$$
\begin{aligned}
C_{B T C A} & =2^{2 N}\left(\frac{1}{2}+\frac{1}{2^{2}}+\cdots+\frac{1}{2^{2 N}}\right) \\
& =2^{2 N}\left(1-\frac{1}{2^{2 N}}\right) \\
& =2^{2 N}-1 .
\end{aligned}
$$

We can find that $C_{B T O T}<C_{B T C A}$, which means that BTOT does not increase the complexity for constructing the binary tree, but decreases a little.

After constructing the binary tree, we need to traverse the tree for each bit-plane. According to Algorithm 1, we can find that the coding process of BTCA and that of BTOT are similar, except that BTOT records some truncation points. However, in order to perform rate distortion optimization, we have to do some extra coding process for each blocks. On the other hand, after recoding the truncation points, we need to apply PCRD optimization to get a final truncation point in each block at a specified bit rate. So, BTOT will take more encoding process than BTCA. Considering BTOT requires much less memory and provides resolution and SNR scalability, the extra coding process is valuable.

In fact, most of the computation cost in entropy coding [26]. For instance, the coding time for SPIHT with arithmetic coding is $0.33 \mathrm{~s}$, while it is $0.14 \mathrm{~s}$ without arithmetic coding [5]. So the methods using entropy coding, such as EBCOT [8], JPEG2000 [9], are too complex to be a standard for space-borne tasks.

\subsection{Memory requirement}

Most of coding methods need to store significant/insignificant coefficients and sets. For example, when coding an image of size $512 \times 512$ at $0.5 \mathrm{bpp}$ for SPIHT, there are about 25000 significant coefficients, 50000 insignificant coefficients, and 32000 insignificant sets. If we use linked list to store them, then each one needs 8 bytes. Hence, the memory for recoding significant/insignificant coefficients and sets is about $0.4 \mathrm{M}$ bytes for SPIHT. Besides, it needs to store the original wavelet image, whose size is $1 \mathrm{M}$ bytes, and needs to store the final coding stream which needs $0.0625 \mathrm{M}$ bytes, so the total memory requirement is about $1.5 \mathrm{M}$ for SPIHT.

If the block size of BTOT is set to $64 \times 64$, then there are $2 \times 64 \times 64$ elements in the binary tree for each block of BTOT, which needs about $0.03 \mathrm{M}$ bytes with each element 4 bytes. Besides, it needs to store the final coding stream and some truncation points which need about $0.063 \mathrm{M}$ bytes, so the total memory requirement for BTOT is about $0.1 \mathrm{M}$ bytes. For BTCA, there are $2 \times 512 \times 512$ elements in the binary tree which needs $2 \mathrm{M}$ bytes, so the total memory requirement for BTCA is about $2.07 \mathrm{M}$ bytes. We can find that the memory requirement for BTOT is much less than that for BTCA.

\subsection{The features of the coding stream}

BTCA provides the quality scalability, because BT$\mathrm{CA}$ is an embedded coding method and it can stop coding or decoding at any specified rate. However, BTCA cannot provide the random access property, because the decoder of BTCA has to decode each region in turn. On the contrary, BTOT not only provides the quality scalability, but also has the random access property. Because BTOT exports the code length of each block, 
the decoder of BTOT can decode any specified blocks. Moreover, if we restrict each block within a wavelet subband, then BTOT can provide resolution scalability, which means the decoder can decode any specified resolution.

\section{Experimental results}

In this section, we conduct some experiments on three remote sensing image sets: the USC-SIPI Image Database[35], the Landsat images[36] and the CCSD$\mathrm{S}$ image test corpus[37], to evaluate the performance of the proposed BTOT method. The USC-SIPI image database contains four volumes. The Aerials volume of the database consists of 38 images. We select three images, i.e., San Diego, North Island NAS of San Diego and Pentagon, for experiment, as shown in Figure $4(a)(b)(c)$. The Landsat images were produced by the Landsat-7 flight, including eight spectral bands and one panchromatic band. Experiments are conducted on two images: Hills and Fires, as shown in Figure 4(d)(e). The CCSDS reference test image set includes a variety of space imaging instrument data such as solar, stellar, planetary, earth observations, etc. We use the Lunar image for experiment, as shown in Figure 4(f).

The images for experiment include different kinds of remote sensing images. All these images are converted into gray ones and size of $512 \times 512 \times 8$ before compression. In addition, we use 9/7-tap biorthogonal wavelet filters [38] for wavelet transform. The size of each block of the proposed BTOT method is $64 \times 64$. The PeakSignal-to-Noise-Ratios (PSNR) for different Compression Ratios (CR) of our method are compared to those of SPIHT [5], SPECK [6], CCSDS [12], JPEG2000 [11], QTC[39], BTCA [26], BTCAS [26] and HAS [28]. The MATLAB source code for BTCA is available ${ }^{1}$, and the source code for BTOT will also be published there if this paper is accepted.

The PSNR and CR are expressed by the following relations [40]:

$$
\begin{gathered}
P S N R(d B)=10 \log _{10} \frac{255^{2}}{M S E}, \\
M S E=\frac{\sum_{i=1}^{W} \sum_{j=1}^{H}\left(x_{i j}-y_{i j}\right)^{2}}{W \times H}, \\
C R(b p p)=\frac{\text { number of coded bits }}{W \times H},
\end{gathered}
$$

${ }^{1}$ MATLAB code: http://www.mathworks.com/matlabcentral/profile/aut ke-kun-huang/ where $x$ and $y$ denote the original image and reconstructed one, respectively, and the images are of size $W \times H$.

Besides PSNR, we also use the Structural Similarity Index Measure (SSIM) [41] and Visual Information Fidelity (VIF) [42] criterion to evaluate the performance, which are considered to be correlated with the quality perception of the human visual system. The SSIM is defined as:

$$
\begin{gathered}
S S I M=l(x, y) c(x, y) s(x, y), \\
l(x, y)=\frac{2 \mu_{x} \mu_{y}+C_{1}}{\mu_{x}^{2} \mu_{y}^{2}+C_{1}}, \\
c(x, y)=\frac{2 \sigma_{x} \sigma_{y}+C_{2}}{\sigma_{x}^{2} \sigma_{y}^{2}+C_{2}}, \\
s(x, y)=\frac{\sigma_{x y}+C_{3}}{\sigma_{x} \sigma_{y}+C_{3}},
\end{gathered}
$$

where the detail parameters can be found in [41].

The model of VIF is defined as follows.

$$
\begin{gathered}
Y(i, k)=\beta(i, k) X(i, k)+V(i, k)+W(i, k) \\
\chi(i, k)=X(i, k)+W(i, k)
\end{gathered}
$$

where $X(i, k)$ is the original image, $\chi(i, k)$ is the image captured by human, $W(i, k)$ is the noise of human vision system and $Y(i, k)$ is the distorted image. $X(i, k)$ is decomposed into several subbands $X_{1}(i, k), X_{2}(i, k), \cdots, X_{m}(i, k)$ by wavelet transformation. Suppose the variance of $W(i, k)$ is $\sigma_{W}, c=0.01$ and $Z(i, k)^{2}=\frac{1}{m} \sum_{j=1}^{M} X_{j}(i, k)^{2}$, then

$$
V I F=\frac{\log _{2}\left(\frac{\beta(i, k)^{2} Z(i, k)^{2}+\sigma_{W}+c}{\sigma_{W}+c}\right)}{\log _{2}\left(\frac{Z(i, k)^{2}+c}{c}\right)} .
$$

For the proposed method, there is only one parameter, i.e., the block size, need to select. Table 3 shows the mean PSNRs of the proposed BTOT method at five bit rates for different block sizes, where the PSNR at each bit rate is the mean value for six testing image. We can find that the performance for the block size $16 \times 16$ is always the lowest. When the block size is too smal1 , there will be many continuous insignificant block, so the compression performance is limited. The results for other block sizes are similar. Considering the position scalability, we select the block size $64 \times 64$ for the proposed method.

Table 4 lists the PSNRs of the proposed method and other algorithms at five bit rates for different remote senssing jumages. From the results, we can draw the following conclusions: 
Table 3: The mean PSNRs of the proposed BTOT method at five bit rates for different block sizes.

\begin{tabular}{cccccc}
\hline Block size & \multicolumn{5}{c}{ PSNR } \\
\cline { 2 - 6 } & 1 bpp & 0.5 bpp & 0.25 bpp & 0.125 bpp & $0.0625 b p p$ \\
\hline $16 \times 16$ & 32.29 & 28.79 & 26.32 & 24.50 & 22.88 \\
$32 \times 32$ & $\mathbf{3 2 . 4 4}$ & 28.96 & 26.52 & 24.74 & 23.41 \\
$\mathbf{6 4} \times \mathbf{6 4}$ & 32.43 & $\mathbf{2 8 . 9 7}$ & 26.56 & $\mathbf{2 4 . 7 9}$ & $\mathbf{2 3 . 4 4}$ \\
$128 \times 128$ & 32.43 & 28.92 & $\mathbf{2 6 . 7 2}$ & 24.78 & 23.35 \\
\hline
\end{tabular}

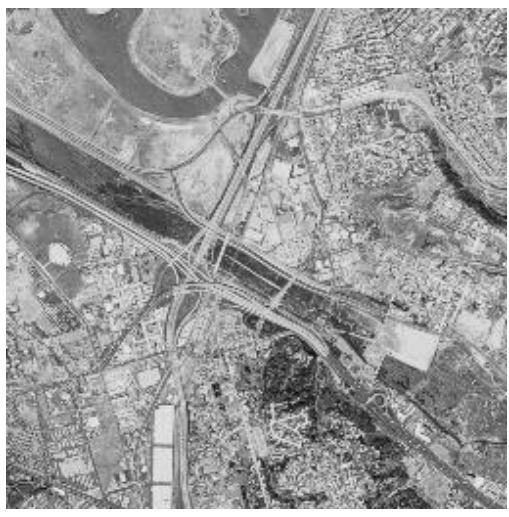

(a) San Diego

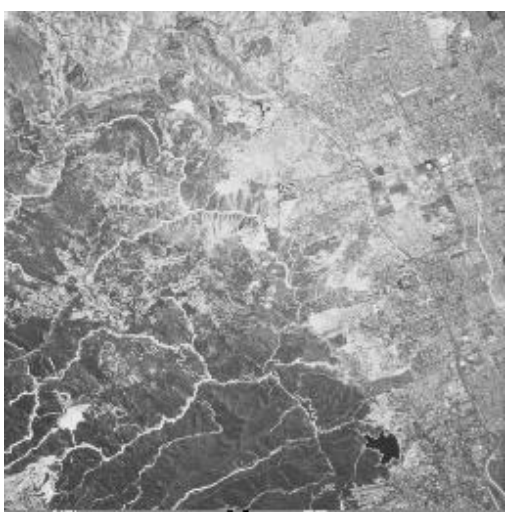

(d) Hills

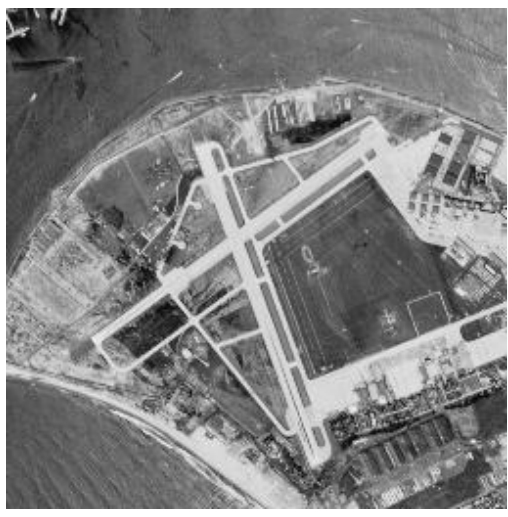

(b) North Island NAS of SanDiego

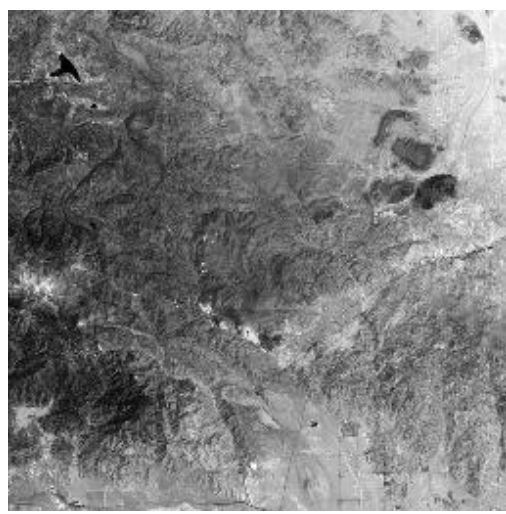

(e) Fires

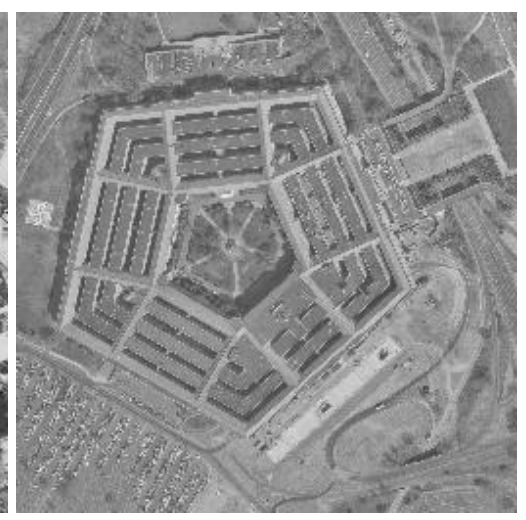

(c) Pentagon

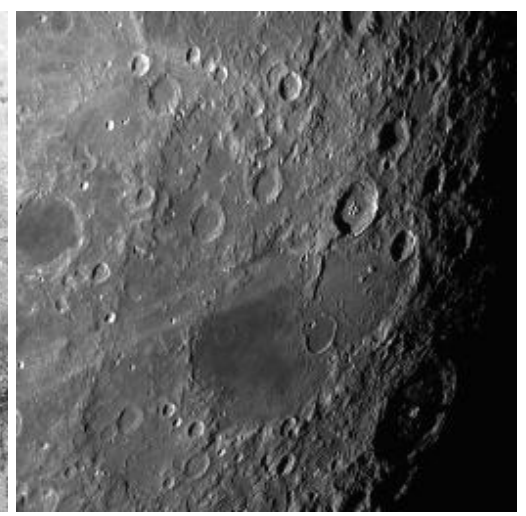

(f) Lunar

Figure 4: Remote sensing images for experiment. 
Table 4: PSNRs of the proposed BTOT method and other algorithms at five bit rates for different remote sensing images.

\begin{tabular}{|c|c|c|c|c|c|c|}
\hline \multirow[t]{2}{*}{ Image } & \multirow[t]{2}{*}{ Algorithm } & \multicolumn{5}{|c|}{ PSNR } \\
\hline & & $1 \mathrm{bpp}$ & $0.5 b p p$ & $0.25 \mathrm{bpp}$ & $0.125 \mathrm{bpp}$ & $0.0625 \mathrm{bpp}$ \\
\hline \multirow[t]{9}{*}{ SanDiego } & SPIHT & 26.61 & 23.37 & 21.40 & 20.08 & 18.89 \\
\hline & SPECK & 26.73 & 23.57 & 21.80 & 20.44 & 19.45 \\
\hline & CCSDS & 26.58 & 23.42 & 21.42 & 19.67 & 14.71 \\
\hline & JPEG2000 & 27.07 & 23.68 & 21.84 & 20.39 & 19.23 \\
\hline & QTC & 26.83 & 23.63 & 21.83 & 20.45 & 19.45 \\
\hline & BTCA & 26.93 & 23.76 & 21.87 & 20.53 & 19.56 \\
\hline & BTCAS & 26.76 & 23.64 & 21.87 & 20.48 & 19.54 \\
\hline & HAS & 26.93 & 23.79 & 21.88 & 20.50 & 19.52 \\
\hline & BTOT & 27.09 & 24.00 & 21.97 & 20.76 & 19.82 \\
\hline \multirow[t]{9}{*}{ NorthSanDiego } & SPIHT & 33.44 & 29.70 & 26.76 & 24.18 & 21.82 \\
\hline & SPECK & 33.45 & 29.76 & 27.02 & 24.84 & 23.03 \\
\hline & CCSDS & 33.21 & 29.48 & 26.46 & 22.70 & 15.54 \\
\hline & JPEG2000 & 33.81 & 29.92 & 27.01 & 24.69 & 22.46 \\
\hline & QTC & 33.60 & 29.86 & 27.10 & 24.89 & 23.05 \\
\hline & BTCA & 33.84 & 30.16 & 27.39 & 25.09 & 23.25 \\
\hline & BTCAS & 33.47 & 29.81 & 27.07 & 24.82 & 23.03 \\
\hline & HAS & 33.80 & 30.42 & 27.38 & 25.06 & 23.22 \\
\hline & BTOT & 33.95 & 30.17 & 27.38 & 25.13 & 23.26 \\
\hline \multirow[t]{9}{*}{ Pentagon } & SPIHT & 34.45 & 30.64 & 28.01 & 26.04 & 24.10 \\
\hline & SPECK & 34.39 & 30.70 & 28.44 & 26.65 & 25.20 \\
\hline & CCSDS & 34.47 & 30.60 & 28.04 & 25.36 & 18.17 \\
\hline & JPEG2000 & 35.08 & 31.11 & 28.54 & 26.57 & 24.73 \\
\hline & QTC & 34.54 & 30.79 & 28.49 & 26.67 & 25.17 \\
\hline & BTCA & 34.85 & 31.09 & 28.60 & 26.80 & 25.37 \\
\hline & BTCAS & 34.81 & 31.02 & 28.58 & 26.70 & 25.21 \\
\hline & HAS & 34.96 & 31.13 & 28.60 & 26.81 & 25.27 \\
\hline & BTOT & 34.86 & 31.08 & 28.67 & 27.06 & 25.43 \\
\hline \multirow[t]{9}{*}{ Hills } & SPIHT & 30.08 & 27.08 & 25.01 & 23.21 & 21.68 \\
\hline & SPECK & 30.19 & 27.39 & 25.27 & 23.67 & 22.49 \\
\hline & CCSDS & 30.34 & 27.40 & 25.13 & 22.88 & 17.78 \\
\hline & JPEG2000 & 30.91 & 27.60 & 25.32 & 23.62 & 22.22 \\
\hline & QTC & 30.36 & 27.49 & 25.31 & 23.71 & 22.50 \\
\hline & BTCA & 30.58 & 27.57 & 25.44 & 23.86 & 22.60 \\
\hline & BTCAS & 30.50 & 27.54 & 25.39 & 23.78 & 22.49 \\
\hline & HAS & 30.51 & 27.59 & 25.58 & 23.83 & 22.55 \\
\hline & BTOT & 30.63 & 27.77 & 25.72 & 23.95 & 22.60 \\
\hline \multirow[t]{9}{*}{ Fires } & SPIHT & 31.25 & 28.38 & 26.17 & 24.59 & 23.22 \\
\hline & SPECK & 31.44 & 28.48 & 26.50 & 25.01 & 23.95 \\
\hline & CCSDS & 31.42 & 28.44 & 26.34 & 24.46 & 19.88 \\
\hline & JPEG2000 & 31.91 & 28.63 & 26.58 & 24.92 & 23.71 \\
\hline & QTC & 31.53 & 28.56 & 26.55 & 25.08 & 23.97 \\
\hline & BTCA & 31.65 & 28.67 & 26.68 & 25.17 & 24.10 \\
\hline & BTCAS & 31.61 & 28.60 & 26.60 & 25.14 & 24.01 \\
\hline & HAS & 31.61 & 28.70 & 26.67 & 25.18 & 24.02 \\
\hline & BTOT & 31.77 & 29.02 & 26.85 & 25.22 & 24.12 \\
\hline \multirow[t]{9}{*}{ Lunar } & SPIHT & 35.56 & 31.13 & 28.04 & 25.48 & 23.28 \\
\hline & SPECK & 35.63 & 31.30 & 28.54 & 26.26 & 24.49 \\
\hline & CCSDS & 35.46 & 30.98 & 27.79 & 24.17 & 14.71 \\
\hline & JPEG2000 & 36.34 & 31.75 & 28.64 & 26.10 & 24.12 \\
\hline & QTC & 35.88 & 31.42 & 28.61 & 26.46 & 24.54 \\
\hline & BTCA & 36.15 & 31.73 & 28.78 & 26.56 & 24.74 \\
\hline & BTCAS & 36.00 & 31.62 & 28.67 & 26.42 & 24.55 \\
\hline & HAS & 36.08 & 31.76 & 28.77 & 26.51 & 24.70 \\
\hline & BTOT & 36.25 & 31.76 & 28.78 & 26.61 & 25.42 \\
\hline
\end{tabular}


Table 5: SSIMs of the proposed BTOT method and other algorithms at five bit rates for different remote sensing images.

\begin{tabular}{|c|c|c|c|c|c|c|}
\hline \multirow[t]{2}{*}{ Image } & \multirow[t]{2}{*}{ Algorithm } & \multicolumn{5}{|c|}{ SSIM } \\
\hline & & $1 \mathrm{bpp}$ & $0.5 \mathrm{bpp}$ & $0.25 b p p$ & $0.125 b p p$ & 0.0625 bpp \\
\hline \multirow[t]{6}{*}{ SanDiego } & SPIHT & 0.9601 & 0.9130 & 0.8578 & 0.7945 & 0.7104 \\
\hline & CCSDS & 0.9600 & 0.9140 & 0.8569 & 0.7648 & 0.4152 \\
\hline & BTCA & 0.9631 & 0.9213 & 0.8746 & 0.8190 & 0.7643 \\
\hline & BTCAS & 0.9616 & 0.9183 & 0.8716 & 0.8153 & 0.7591 \\
\hline & HAS & 0.9635 & 0.9211 & 0.8731 & 0.8202 & 0.7606 \\
\hline & BTOT & 0.9645 & 0.9256 & 0.8748 & 0.8296 & 0.7770 \\
\hline \multirow[t]{6}{*}{ Hills } & SPIHT & 0.9813 & 0.9623 & 0.9375 & 0.9030 & 0.8575 \\
\hline & CCSDS & 0.9824 & 0.9648 & 0.9392 & 0.8931 & 0.6929 \\
\hline & BTCA & 0.9833 & 0.9661 & 0.9438 & 0.9178 & 0.8864 \\
\hline & BTCAS & 0.9829 & 0.9658 & 0.9429 & 0.9149 & 0.8832 \\
\hline & HAS & 0.9830 & 0.9664 & 0.9454 & 0.9166 & 0.8858 \\
\hline & BTOT & 0.9835 & 0.9676 & 0.9474 & 0.9184 & 0.8862 \\
\hline \multirow[t]{6}{*}{ Fires } & SPIHT & 0.9894 & 0.9771 & 0.9650 & 0.9489 & 0.9282 \\
\hline & CCSDS & 0.9897 & 0.9794 & 0.9662 & 0.9468 & 0.8503 \\
\hline & BTCA & 0.9890 & 0.9805 & 0.9689 & 0.9554 & 0.9422 \\
\hline & BTCAS & 0.9901 & 0.9802 & 0.9682 & 0.9550 & 0.9408 \\
\hline & HAS & 0.9902 & 0.9807 & 0.9688 & 0.9557 & 0.9411 \\
\hline & BTOT & 0.9905 & 0.9820 & 0.9700 & 0.9559 & 0.9421 \\
\hline \multirow[t]{6}{*}{ Lunar } & SPIHT & 0.9960 & 0.9890 & 0.9773 & 0.9585 & 0.9296 \\
\hline & CCSDS & 0.9959 & 0.9885 & 0.9758 & 0.9424 & 0.5812 \\
\hline & BTCA & 0.9965 & 0.9904 & 0.9809 & 0.9677 & 0.9505 \\
\hline & BTCAS & 0.9964 & 0.9901 & 0.9803 & 0.9666 & 0.9479 \\
\hline & HAS & 0.9965 & 0.9904 & 0.9809 & 0.9675 & 0.9503 \\
\hline & BTOT & 0.9966 & 0.9905 & 0.9808 & 0.9681 & 0.9580 \\
\hline \multirow[t]{6}{*}{ Average } & SPIHT & 0.9817 & 0.9604 & 0.9344 & 0.9012 & 0.8564 \\
\hline & CCSDS & 0.9820 & 0.9617 & 0.9345 & 0.8868 & 0.6349 \\
\hline & BTCA & 0.9830 & 0.9646 & 0.9421 & 0.9150 & 0.8858 \\
\hline & BTCAS & 0.9828 & 0.9636 & 0.9407 & 0.9130 & 0.8827 \\
\hline & HAS & 0.9833 & 0.9647 & 0.9421 & 0.9150 & 0.8845 \\
\hline & BTOT & 0.9838 & 0.9664 & 0.9433 & 0.9180 & 0.8908 \\
\hline
\end{tabular}


Table 6: VIFs of the proposed BTOT method and other algorithms at five bit rates for different remote sensing images.

\begin{tabular}{ccccccc}
\hline \multirow{2}{*}{ Image } & \multirow{2}{*}{ Algorithm } & \multicolumn{5}{c}{ VIF } \\
\cline { 3 - 6 } & & lbpp & 0.5 bpp & 0.25 bpp & 0.125 bpp & $0.0625 b p p$ \\
\hline SanDiego & BTCA & 0.4481 & 0.3006 & 0.1910 & 0.1163 & 0.0683 \\
& BTCAS & 0.4554 & 0.2990 & 0.1891 & 0.1118 & 0.0652 \\
& HAS & 0.4525 & 0.2954 & $\mathbf{0 . 1 9 3 7}$ & 0.1155 & 0.0638 \\
\multirow{5}{*}{ Fires } & BTOT & $\mathbf{0 . 4 6 3 5}$ & $\mathbf{0 . 3 1 1 5}$ & 0.1921 & $\mathbf{0 . 1 3 4 6}$ & $\mathbf{0 . 0 8 3 3}$ \\
& BTCA & 0.5683 & 0.3841 & 0.2420 & 0.1419 & 0.0816 \\
& BTCAS & 0.5793 & 0.3862 & 0.2441 & 0.1437 & 0.0799 \\
& HAS & $\mathbf{0 . 5 8 4 7}$ & 0.3840 & 0.2444 & $\mathbf{0 . 1 4 8 3}$ & 0.0806 \\
Lunar & BTOT & 0.5789 & $\mathbf{0 . 4 1 0 6}$ & $\mathbf{0 . 2 5 2 0}$ & 0.1480 & $\mathbf{0 . 0 8 3 1}$ \\
& BTCA & 0.7226 & 0.4728 & 0.3087 & 0.1817 & 0.1046 \\
& BTCAS & 0.7139 & 0.4753 & 0.3040 & 0.1810 & 0.1021 \\
& HAS & $\mathbf{0 . 7 2 3 5}$ & $\mathbf{0 . 4 7 9 4}$ & 0.3083 & 0.1856 & 0.1086 \\
& BTOT & $\mathbf{0 . 7 2 3 5}$ & 0.4768 & $\mathbf{0 . 3 0 9 8}$ & $\mathbf{0 . 1 9 0 1}$ & $\mathbf{0 . 1 6 7 7}$ \\
\hline
\end{tabular}

- CCSDS almost attains the worst results for each image and each bit rate, because it specifically targets onboard mission and focuses more on complexity and less on compression performance.

- JPEG2000 in general achieves better performance than SPIHT or SPECK, especially at higher bit rates. However, JPEG2000 is too complex to be a standard for airborne missions.

- BTCA gets better performance than QTC, which proves that binary tree provides a more efficien$t$ image representation than quadtree.

- The PSNR of BTCAS is lower than that of BTCA, though BTCAS provides random access property and requires less memory.

- HAS generates an importance weighting mask according to the human visual characteristics before applying BTCA, but HAS cannot improve the PSNR compared with BTCA. The PSNRs of both algorithms are very similar.

- The proposed BTOT achieves the highest performance, especially significantly improves the performance compared with BTCA. For SanDiego image, the PSNR of BTOT increases an average of $0.64 \mathrm{~dB}, 0.31 \mathrm{~dB}, 1.55 \mathrm{~dB}, 0.27 \mathrm{~dB}, 0.27 \mathrm{~dB}, 0.18 \mathrm{~d}-$ B, $0.25 \mathrm{~dB}, 0.19 \mathrm{~dB}$ than SPIHT, SPECK, CCSDS, JPEG2000, QTC, BTCA, BTCAS and HAS, respectively.

Table 5 lists the SSIMs of the proposed method and other algorithms. We can find that the SSIM results are similar to the PSNR results. At the same compression ratio, the higher the PSNR is, the better the SSIM usually is. For example, at $0.5 \mathrm{bpp}$ for SanDiego image, the PSNR of BTOT is 24.00, which is the highest of al1 methods, and the SSIM is 0.9256 , which is also the highest of all methods. The average SSIM of all images for each method at each bit rate is listed at the end of Table 5. It can be seen that the average of the proposed method is the higest of all methods at each bit rate.

Table 6 lists the VIFs of the proposed method and other algorithms. Except sometimes the VIF of HAS is the better, the performance of the proposed method is the best, which proves that HAS is a state-of-the-art method and the proposed method is the better.

Figure 5 shows the comparison of two compression algorithms with the image of North Island NAS of San Diego at $0.25 \mathrm{bpp}$. The number of coded coefficients with BTOT is 12584 , while it is 11627 with SPIHT. The reconstructed image with BTOT is clearer than that with SPIHT in the red rectangles. Figure 6 gets a similar result with the Pentagon image.

Table 7 shows the comparison of PSNR, SSIM, complexity, memory requirement, coding time and other properties for different methods at the same compression ratio. SPIHT is implemented without arithmetic coding [43]. The program of CCSDS can be download from [44]. The programs are evaluated on a computer with Intel Core i7-4910MQ CPU and 32 G memory. The PSNR, SSIM and coding time are the corresponding mean values for six testing images, respectively. We can find that the PSNR of BTOT is the highest, so does the SSIM. According to Eq. (7) and Eq. (8), the complexity of BTCA and BTOT is proportional to the num- 


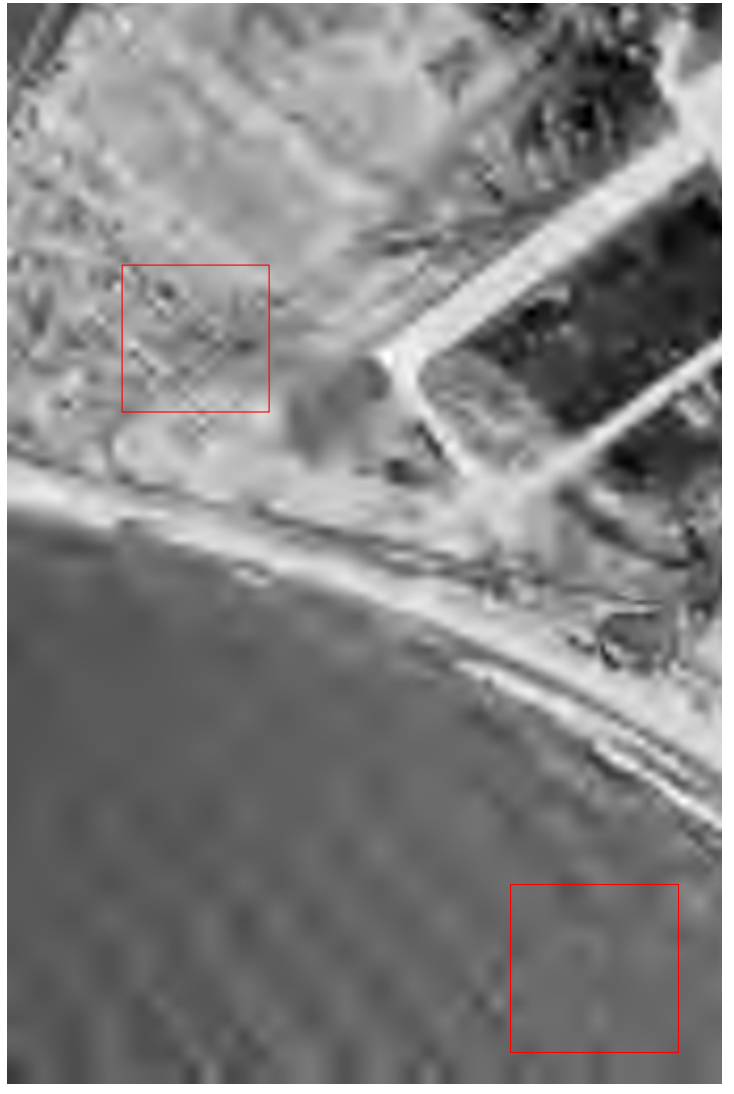

(a) Reconstructed image with BTOT

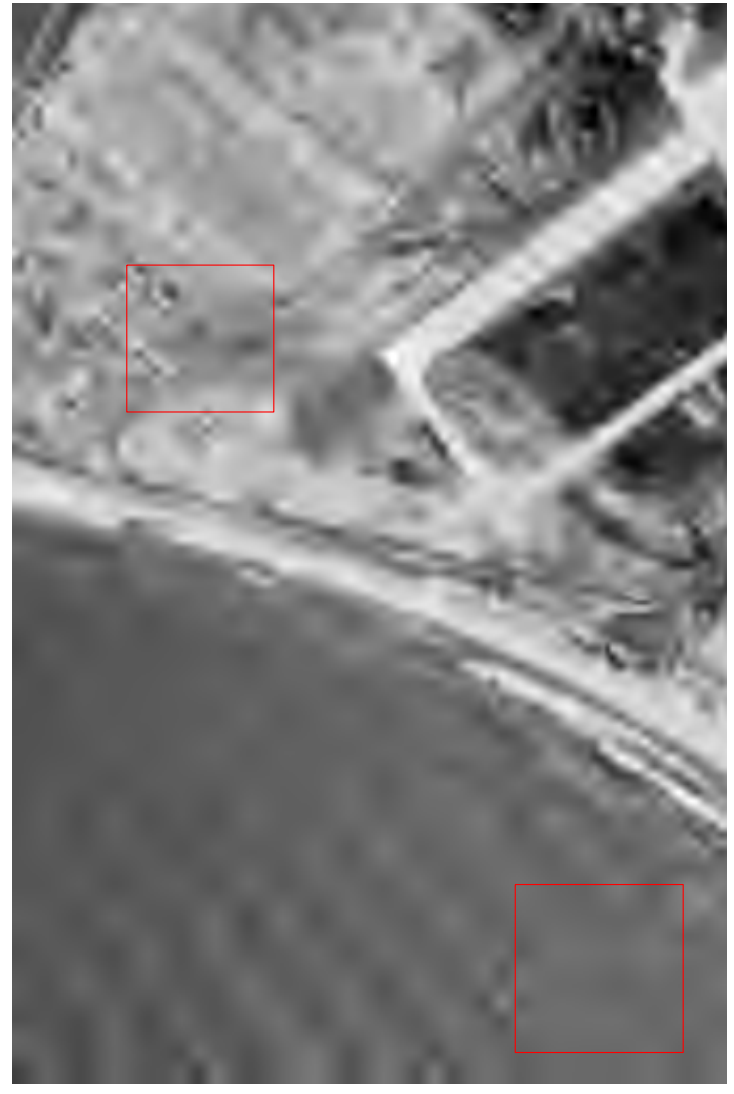

(b) Reconstructed image with SPIHT

Figure 5: Comparison of two compression algorithms with the image of North Island NAS of San Diego at 0.25bpp. The number of coded coefficients with BTOT is 12584, while it is 11627 with SPIHT. The reconstructed image with BTOT is clearer than that with SPIHT. For example, there are more textures in the red rectangles of the reconstructed image with BTOT. 


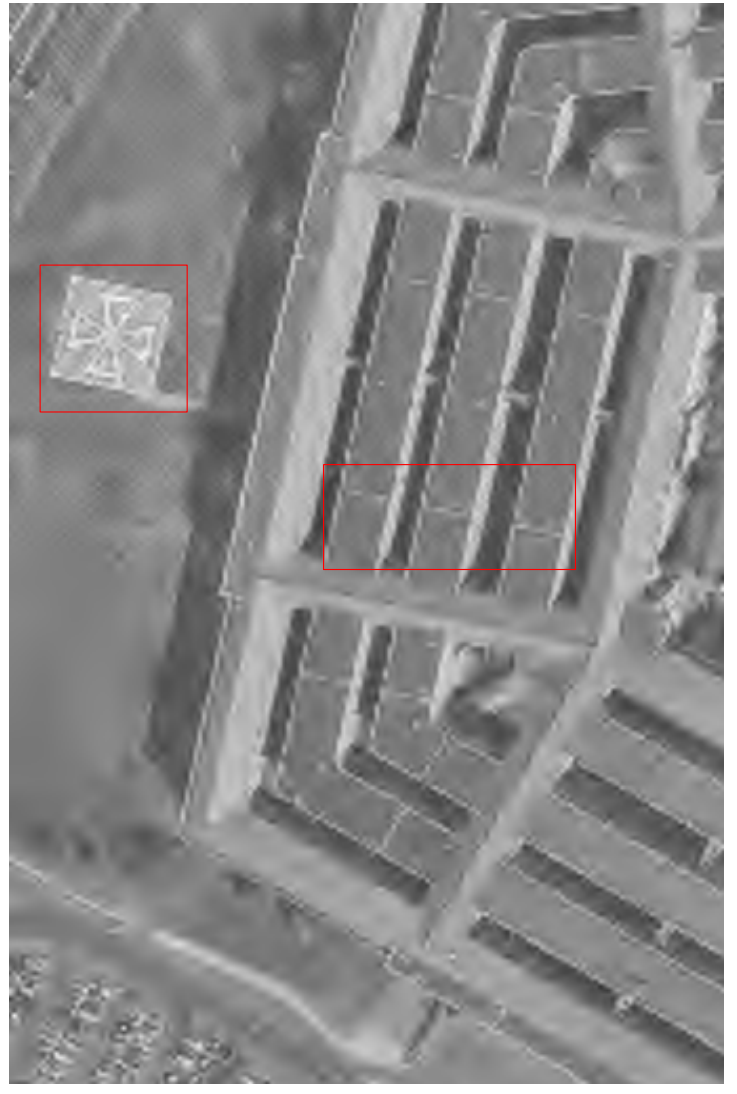

(a) Reconstructed image with BTOT

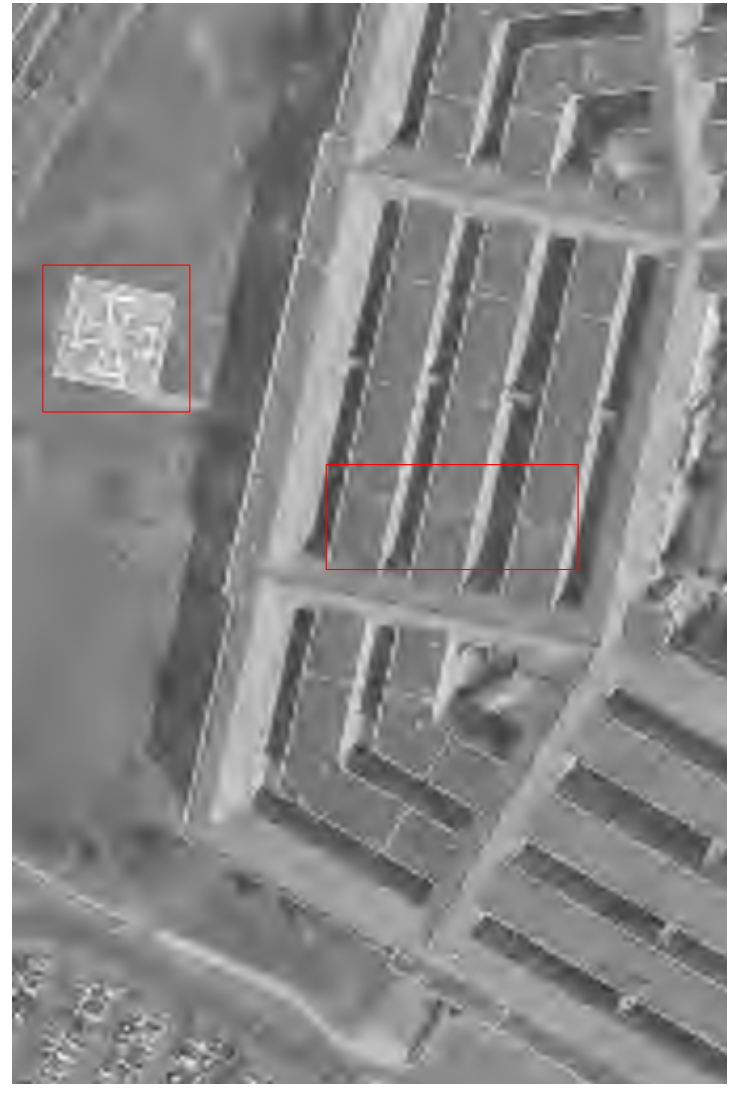

(b) Reconstructed image with SPIHT

Figure 6: Comparison of two compression algorithms with Pentagon at $0.5 \mathrm{bpp}$. The number of coded coefficients with BTOT is 27452, while it is 19548 with SPIHT. The reconstructed image with BTOT is clearer than that with SPIHT. For example, the lines of the reconstructed image with BTOT are more clearer in the right rectangles. 
Table 7: Comparison of different methods at the same compression ratio.

\begin{tabular}{ccccc}
\hline Method & SPIHT & CCSDS & BTCA & BTOT \\
\hline CR & $0.5 \mathrm{bpp}$ & $0.5 \mathrm{bpp}$ & $0.5 \mathrm{bpp}$ & $0.5 \mathrm{bpp}$ \\
PSNR & 28.38 & 28.39 & 28.83 & 28.97 \\
SSIM & 0.9639 & 0.9645 & 0.9677 & 0.9856 \\
Complexity & $O(n)$ & $O(n)$ & $O(n)$ & $O(n)$ \\
Memory requirement & $1.5 \mathrm{M}$ & $1.1 \mathrm{M}$ & $2 \mathrm{M}$ & $0.1 \mathrm{M}$ \\
Coding time & 0.10 & 0.75 & 0.11 & 0.18 \\
Quality scalability & yes & yes & yes & yes \\
Position scalability & no & no & no & yes \\
Resolution scalability & no & no & no & yes
\end{tabular}

ber of image pixels $n$, so do those of SPIHT and CCSDS.BTOT is only a little slower than SPIHT, but is much faster than CCSDS, because CCSDS uses entropy coding. Based on the analysis in section 4.2 , the memory requirement for BTOT is much less than that for BTCA. Moreover, BTOT provides quality, position and resolution scalability, while SPIHT, CCSDS and BTCA only provide quality scalability.

\section{Conclusion}

In this paper, we have proposed an efficient compression method for remote sensing image. We first divide the entire wavelet image to several blocks and encode them individually by BTCA, then apply rate-distortion optimization to get higher compression ratio and lower memory requirement, as well as the random access property. The proposed method is simple and easy to be implemented, so the proposed method is very suitable for remote sensing image. Furthermore, we can use wavelet packet transform to construct binary tree, design entropy coding and parallel algorithm to improve efficiency, which are our future works.

\section{References}

[1] A. Gunes, M. B. Guldogan, Joint underwater target detection and tracking with the bernoulli filter using an acoustic vector sensor, Digital Signal Processing 48 (1) (2016) 246 - 258.

[2] S. Uur, O. Arkan, A. C. Grbz, SAR image reconstruction by expectation maximization based matching pursuit, Digital Signal Processing 37 (2) (2015) 75 - 84.

[3] M. M. Nielsen, Remote sensing for urban planning and management: The use of window-independent context segmentation to extract urban features in stockholm, Computers, Environment and Urban Systems 52 (7) (2015) 1-9.

[4] J. M. Shapiro, Embedded image coding using zerotrees of wavelet coefficients, IEEE Transations on Signal Processing 41 (12) (1993) 3445-3462.
[5] A. Said, W. A. Pearlman, A new, fast, and efficient image codec based on set partitioning in hierarchical trees, IEEE Transactions on Circuits and Systems for Video Technology 6 (3) (1996) 243-250.

[6] W. A. Pearlman, A. Islam, N. Nagaraj, A. Said, Efficient, low-complexity image coding with a set-partitioning embedded block coder, IEEE Transactions on Circuits and Systems for Video Technology 14 (11) (2004) 1219-1235.

[7] A. Munteanu, J. Cornelis, G. V. D. Auwera, P. Cristea, Wavelet image compression - the quadtree coding approach, IEEE Transactions on Information Technology in Biomedicine 3 (3) (1999) 176-185.

[8] D. Taubman, High performance scalable image compression with EBCOT, IEEE Transations on Image Processing 9 (7) (2000) 1158-1170.

[9] D. Taubman, M. Marcellin, JPEG2000 Image Compression Fundamentals, Standards and Practice: Image Compression Fundamentals, Standards and Practice, Vol. 642, Springer Science \& Business Media, 2012.

[10] B. Li, R. Yang, H. X. Jiang, Remote-sensing image compression using two-dimensional oriented wavelet transform, IEEE Transactions on Geoscience and Remote Sensing 49 (1) (2011) 236-250.

[11] P. Kulkarni, A. Bilgin, M. W. Marcellin, J. C. Dagher, J. H. Kasner, T. J. Flohr, J. C. Rountree, Hyperspectral Data Compression, Springer US, Boston, MA, 2006, Ch. Compression of Earth Science Data with JPEG2000, pp. 347-378.

[12] [Online], Image data compression, Nov. 2005. ser. blue book, CCSDS 122.0-b-1, http: //public.ccsds.org/publications/BlueBooks.aspx.

[13] [Online], Consultative committee for space data systems, http: / / www. ccsds.org.

[14] F. Garcia-Vilchez, J. Serra-Sagrista, Extending the CCSDS recommendation for image data compression for remote sensing scenarios, IEEE Transactions on Geoscience and Remote Sensing 47 (10) (2009) 3431-3445.

[15] X. S. Hou, J. Yang, G. F. Jiang, X. M. Qian, Complex sar image compression based on directional lifting wavelet transfor$\mathrm{m}$ with high clustering capability, IEEE Transactions on Geoscience and Remote Sensing 51 (1) (2013) 527-538.

[16] H. ZainEldin, M. A. Elhosseini, H. A. Ali, A modified listless strip based SPIHT for wireless multimedia sensor networks, Computers and Electrical Engineeringdoi:http://dx.doi.org/10.1016/j.compeleceng.2015.10.001.

[17] V. B. Raju, K. J. Sankar, C. D. Naidu, S. Bachu, Multispectral image compression for various band images with high resolution improved DWT SPIHT, International Journal of Signal Processing, Image Processing and Pattern Recognition 9 (2) (2016) 271-286.

[18] X. L. Tang, W. A. Pearlman, Hyperspectral Data Compression, Springer US, Boston, MA, 2006, Ch. Three-Dimensional Wavelet-Based Compression of Hyperspectral Images, pp. 273308.

[19] X. S. Hou, M. Han, C. Gong, X. M. Qian, SAR complex image data compression based on quadtree and zerotree coding in discrete wavelet transform domain: A comparative study, Neurocomputing 148 (2015) $561-568$.

[20] M. Gaeta, V. Loia, S. Tomasiello, Cubic bspline fuzzy transforms for an efficient and secure compression in wireless sensor networks, Information Sciences 339 (2016) 19 - 30.

[21] M. Rostami, O. Michailovich, Z. Wang, Gradient-based surface reconstruction using compressed sensing, in: IEEE International Conference on Image Processing (ICIP), 2012, pp. 913-916.

[22] M. Rostami, O. V. Michailovich, Z. Wang, Surface reconstruction in gradient-field domain using compressed sensing, IEEE 
Transactions on Image Processing 24 (5) (2015) 1628-1638.

[23] X. Zhan, R. Zhang, D. Yin, C. Huo, Sar image compression using multiscale dictionary learning and sparse representation, IEEE Geoscience and Remote Sensing Letters 10 (5) (2013) 1090-1094.

[24] W. Fu, S. Li, L. Fang, J. A. Benediktsson, Adaptive spectralspatial compression of hyperspectral image with sparse representation, IEEE Transactions on Geoscience and Remote Sensing PP (99) (2016) 1-12. doi:10.1109/TGRS.2016.2613848.

[25] P. A. M. Oliveira, R. J. Cintra, F. M. Bayer, S. Kulasekera, Lowcomplexity image and video coding based on an approximate discrete tchebichef transform, IEEE Transactions on Circuits and Systems for Video Technology PP (PP) (2016) 1-10.

[26] K. K. Huang, D. Q. Dai, A new on-board image codec based on binary tree with adaptive scanning order in scan-based mode, IEEE Transactions on Geoscience and Remote Sensing 50 (10) (2012) 3737-3750.

[27] H. Liu, K. K. Huang, Embedded image compression based on fractal and wavelet, International Journal of Digital Content Technology and Its Application 8 (2) (2014) 133-139.

[28] C. P. Shi, J. P. Zhang, Y. Zhang, A novel vision-based adaptive scanning for the compression of remote sensing images, IEEE Transactions on Geoscience and Remote Sensing 54 (3) (2016) 1336-1348.

[29] C. P. Shi, J. P. Zhang, Y. Zhang, Content-based onboard compression for remote sensing images, Neurocomputing 191 (2016) 330-340.

[30] S. G. Wang, M. C. Zhou, Z. W. Li, C. Y. Wang, A new modified reachability tree approach and its applications to unbounded Petri nets, IEEE Transactions on Systems, Man, and Cybernetics: Systems 43 (4) (2013) 932-940.

[31] J. T. Liu, C. H. Sui, D. W. Deng, J. W. Wang, B. Feng, W. Y. Liu, C. H. Wu, Representing conditional preference by boosted regression trees for recommendation, Information Sciences 327 (2016) $1-20$.

[32] Y.-L. Chen, C.-C. Wu, K. Tang, Time-constrained cost-sensitive decision tree induction, Information Sciences 354 (2016) 140 152.

[33] E. Shusterman, M. Feder, Image compression via improved quadtree decomposition algorithms, IEEE Transactions on Image Processing 3 (2) (1994) 207-215.

[34] C. A. Shaffer, R. Juvvadi, L. S. Heath, Generalized comparison of quadtree and bintree storage requirements, Image and Vision Computing 11 (7) (1993) 402-412.

[35] [Online], The USC-SIPI image database, http://sipi.usc.edu/database/.

[36] [Online], United states geological survey, landsat project website, http: / / landsat.usgs.gov/.

[37] [Online], Consultative committee for space data systems, CCSDS image test, http://cwe.ccsds.org/sls/docs/sls-dc/.

[38] M. Antonini, M. Barlaud, P. Mathieu, I. Daubechies, Image coding using wavelet transform, IEEE Transations on Image Processing 1 (2) (1992) 205-220.

[39] S. T. Hsiang, J. W. Woods, Embedded image coding using zeroblock of subband/wavelet coefficients and context modeling, in: Data Compression Conf., Washington, DC, 2001, pp. 83-92.

[40] H. Liu, K.-K. Huang, Zerotree wavelet image compression with weighted sub-block-trees and adaptive coding order, International Journal of Wavelets Multiresolution and Information Processing 14 (4) (2016) 1650021.

[41] Z. Wang, A. C. Bovik, H. R. Sheikh, E. P. Simoncelli, Image quality assessment: from error visibility to structural similarity, IEEE Transactions on Image Processing 8 (03) (2004) 600-612.

[42] H. R. Sheikh, A. C. Bovik, Image information and visual quality,
IEEE Transactions on Image Processing 15 (2) (2006) 430-44.

[43] [Online], Center for image processing research, http://ipl.rpi.edu/.

[44] [Online], University of nebraska-lincoln, http://hyperspectral.unl.edu/. 